OPEN ACCESS

Edited by:

Toshinori Sato

Keio University, Japan

Reviewed by:

Ryosuke Suzuki,

National Institute of Infectious

Diseases, Japan

Sonja Best,

National Institute of Allergy

and Infectious Diseases, USA

${ }^{*}$ Correspondence:

Xianzhu Xia

xiaxzh@cae.cn

Songtao Yang

yst62041@163.com:

Hualei Wang

whl831125@163.com

Specialty section: This article was submitted to

Virology,

a section of the journal

Frontiers in Microbiology

Received: 02 February 2016

Accepted: 04 April 2016

Published: 20 April 2016

Citation:

Cao Z, Wang $H$, Wang $L$, Li L, Jin $H$, Xu C, Feng N, Wang J, Li Q, Zhao Y,

Wang T, Gao Y, Lu Y, Yang $S$ and Xia $X$ (2016) Visual Detection

of West Nile Virus Using Reverse

Transcription Loop-Mediated Isothermal Amplification Combined with a Vertical Flow Visualization Strip.

Front. Microbiol. 7:554.

doi: 10.3389/fmicb.2016.00554

\section{Visual Detection of West Nile Virus Using Reverse Transcription Loop-Mediated Isothermal Amplification Combined with a Vertical Flow Visualization Strip}

Zengguo Cao', Hualei Wang ${ }^{1,2 *}$, Lina Wang ${ }^{1,3}$, Ling Li ${ }^{1,4}$, Hongli Jin ${ }^{1,5}$, Changping $X u^{1,6}$, Na Feng',2, Jianzhong Wang ${ }^{1}$, Qian Li', Yongkun Zhao', Tiecheng Wang ${ }^{1}$, Yuwei Gao ${ }^{1,2}$, Yiyu Lu ${ }^{1,6}$, Songtao Yang ${ }^{1,2 *}$ and Xianzhu Xia ${ }^{1,2 *}$

' Key Laboratory of Jilin Province for Zoonosis Prevention and Control, Institute of Military Veterinary, Academy of Military Medical Sciences, Changchun, China, ${ }^{2}$ Jiangsu Co-innovation Center for Prevention and Control of Important Animal Infectious Disease and Zoonoses, Yangzhou, China, ${ }^{3}$ Animal Science and Technology College, Jilin Agricultural University, Changchun, China, ${ }^{4}$ College of Veterinary Medicine, Jilin University, Changchun, China, ${ }^{5}$ Changchun SR Biological Technology Co., Ltd., Changchun, China, ${ }^{6}$ Key Laboratory of Emergency Detection for Public Health of Zhejiang Province, Zhejiang Provincial Center for Disease Control and Prevention, Hangzhou, China

West Nile virus (WNV) causes a severe zoonosis, which can lead to a large number of casualties and considerable economic losses. A rapid and accurate identification method for WNV for use in field laboratories is urgently needed. Here, a method utilizing reverse transcription loop-mediated isothermal amplification combined with a vertical flow visualization strip (RT-LAMP-VF) was developed to detect the envelope (E) gene of WNV. The RT-LAMP-VF assay could detect $10^{2}$ copies/ $\mu$ l of an WNV RNA standard using a 40 min amplification reaction followed by a 2 min incubation of the amplification product on the visualization strip, and no cross-reaction with other closely related members of the Flavivirus genus was observed. The assay was further evaluated using cells and mouse brain tissues infected with a recombinant rabies virus expressing the $E$ protein of WNV. The assay produced sensitivities of $10^{1.5} \mathrm{TCID}_{50} / \mathrm{ml}$ and $10^{1.33} \mathrm{TCID}_{50} / \mathrm{ml}$ for detection of the recombinant virus in the cells and brain tissues, respectively. Overall, the RT-LAMP-VF assay developed in this study is rapid, simple and effective, and it is therefore suitable for clinical application in the field.

Keywords: West Nile virus, visual detection, reverse transcription loop-mediated isothermal amplification, visualization strip, RT-LAMP-VF

\section{INTRODUCTION}

West Nile virus (WNV) infection leads to an acute febrile zoonosis, which can cause disease in birds, humans and horses ${ }^{1}$ (Gubler, 2001). The manifestations of WNV infection include West Nile fever and West Nile encephalitis, which together comprise symptoms ranging from a benign or often symptomless infection to fever and neuroinvasive disease (Gubler, 2001; Sejvar et al., 2003; Hayes and O'Leary, 2004; Hayes and Gubler, 2006). WNV infection gained worldwide attention in

\footnotetext{
${ }^{1}$ http://www.oie.int/en/for-the-media/animal-diseases/animal-disease-information-summaries/
} 
1999, when it was first detected in America during an outbreak in New York City (Nash et al., 2001; Hayes and Gubler, 2006). In America, as of January 12, 2016, a total of 48 states have reported WNV infection in humans, birds, or mosquitoes ${ }^{2}$.

WNV is an arthropod-borne, neurotropic, enveloped Flavivirus with a single-stranded, positive-sense RNA genome and is a member of the Japanese encephalitis virus (JEV) serogroup, which includes JEV, Murray Valley encephalitis virus (MVEV) and St. Louis encephalitis virus (Mukhopadhyay et al., 2003; Solomon et al., 2003; Lim et al., 2011). The RNA genome of WNV is approximately 11,000 nucleotides in length and encodes three structural [capsid (C), premembrane (prM) or membrane $(\mathrm{M})$, and envelope (E)] proteins and seven non-structural (NS1, NS2a, NS2b, NS3, NS4a, NS4b, and NS5) proteins (Deubel et al., 2001; Brinton, 2002; Parida et al., 2004). Phylogenetic analysis of the WNV E protein sequence has indicated that WNV can be separated into two main lineages (lineages 1 and 2) as well as several additional minor lineages (lineage 3 , lineage 4 , lineage 5 and the putative lineage 6; Lanciotti et al., 2002; Vazquez et al., 2010; Del Amo et al., 2013; Faggioni et al., 2014).

There are many laboratory methods for diagnosing WNV, including virus isolation, immunohistochemistry, immunofluorescence, plaque reduction neutralization test (PRNT), enzyme-linked immunosorbent assay (ELISA), reverse transcription PCR (RT-PCR), real-time RT-PCR, and nested RT-PCR, among others (Dauphin and Zientara, 2007; De Filette et al., 2012). Some of these methods have been approved by the American Centers for Disease Control and Prevention (American CDC). However, the ELISA has cross-reactivity with other flaviviruses when used for serodiagnosis. Additionally, the above nucleic acid amplification methods are disadvantageous because they require expensive equipment and are timeconsuming to perform. Furthermore, both virus isolation and PRNT are tedious and technically complex and require over a week to perform. Thus, there is an urgent need to develop a rapid and simple diagnostic method for WNV to improve detection efficiency.

Loop-mediated isothermal amplification (LAMP) is a novel technique that can amplify DNA with high efficiency, specificity and rapidity under isothermal conditions (Notomi et al., 2000). Because this amplification technique requires isothermal conditions, LAMP can be performed using only a simple water bath. Moreover, LAMP can detect RNA templates by using reverse transcriptase together with DNA polymerase for amplification (Whiting and Champoux, 1998; Notomi et al., 2000; Ge et al., 2013). Several RNA viruses have been successfully detected using RT-LAMP assays (Kurosaki et al., 2007; Ge et al., 2013). The gold immunochromatographic assay is another technique that has been widely applied for the detection of various viruses (Mikawa et al., 2009; Wang et al., 2010). Amplicon detection using a vertical flow (VF) visualization strip has been previously applied to accelerate and simplify the process of interpreting LAMP assay results (Cui et al., 2012). In the current study, we developed a RT-LAMP assay coupled with a VF visualization strip for rapid, simple, and accurate visual detection

${ }^{2}$ http://www.cdc.gov/westnile/index.html of WNV. Because of these characteristics, this RT-LAMP-VF assay is useful for field laboratory diagnosis of WNV infection.

\section{MATERIALS AND METHODS}

\section{Viruses and Extraction of Viral RNA}

The recombinant rabies virus SRV9 strain was modified to express the E protein (EP) of WNV (GenBank Accession: DQ211652; rRABV-WNVE) and stored in our laboratory. The recombinant virus was propagated in BSR cells, a clone of the BHK-21 cell line (baby hamster kidney cells), which were grown in Dulbecco's modified Eagle's medium (DMEM, Gibco, Grand Island, NY, USA) supplemented with $5 \%$ fetal bovine serum (FBS, Gibco, Grand Island, NY, USA) at $37^{\circ} \mathrm{C}$ in an incubator. rRABV was titrated in Neuroblastoma (NA) cell as described previously (Wang et al., 2011). Viral RNA was extracted using a commercial RNA extraction kit (RNeasy Mini Kit, Qiagen, Hilden, Germany). All operations were performed according to the manufacturer's instructions.

\section{Preparation of WNV RNA Standards}

Artificial viral RNA encoding a partial WNV E gene sequence (GenBank Accession: DQ211652) was synthesized; this construct was modified via PCR to include the T7 promoter sequence at its $5^{\prime}$ terminus. The PCR products were transcribed in vitro using T7 RNA polymerase (TaKaRa Biotechnology Co., Ltd., Dalian, China) according to the manufacturer's instructions. The RNA transcripts were then purified and quantified, and 10-fold serial dilutions of the RNA ranging from $10^{7}$ to $10^{0}$ copies per $\mu l$ were prepared.

\section{Primer Design}

The complete genome sequences of 30 strains of WNV isolated over various years from different regions and disparate species were analyzed, and the $\mathrm{E}$ gene of $\mathrm{WNV}$ was selected as the target region for the RT-LAMP-VF assay (the alignment of WNV E gene sequences was shown in Figure 1). Six primers, targeting eight distinct regions of the gene, were designed for the assay using the PrimerExplorer V4 program ${ }^{3}$. To detect reaction products by VF, the LF and LB primers were $5^{\prime}$-labeled with biotin and FITC. The details are shown in Figure 2 and Table 1. All primers were synthesized by Sangon Biotech Biotechnology Co., Ltd., (Shanghai, China).

\section{RT-LAMP-VF Reaction and Product Detection}

The RT-LAMP-VF assay was performed using a $25-\mu l$ total reaction volume containing a mixture of $0.4 \mu \mathrm{M}$ of the inner primers FIP and BIP, $0.2 \mu \mathrm{M}$ of the loop primers LF and $\mathrm{LB}, 0.1 \mu \mathrm{M}$ of the outer primers $\mathrm{F} 3$ and B3, $1.4 \mathrm{mM}$ of each deoxynucleoside triphosphate (dNTP), $8 \mathrm{mM} \mathrm{MgSO}_{4}, 0.2 \mathrm{M}$ betaine, $5 \mathrm{U}$ of avian myeloblastosis virus reverse transcriptase (Bioer Technology Co., Ltd., Hangzhou, China), 8 U of Bst DNA

\footnotetext{
${ }^{3}$ http://primerexplorer.jp/elamp4.0.0/index.html
} 


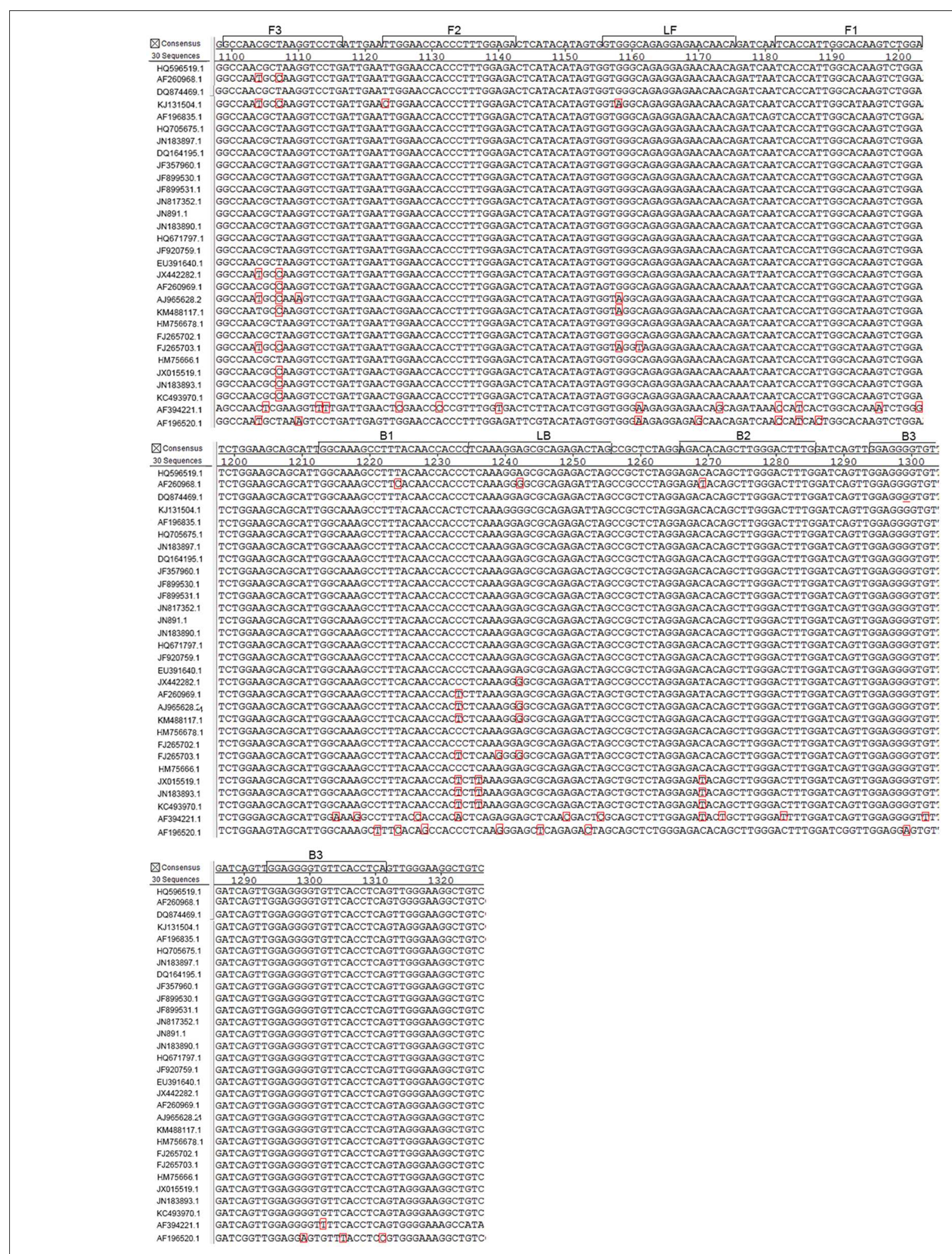

FIGURE 1 | Alignment of West Nile virus (WNV) E gene sequences and positions of the primers used for RT-LAMP. The E gene of WNV was retrieved from GenBank and analyzed using MegAlign software. Primers were designed based on a conserved region (nucleotides 2065-2277 of the genome). The GenBank accession numbers of the aligned strains (from top to bottom) are HQ596519.1, AF260968.1, AF196520, KJ131504.1, AF196835.1, HQ705675.1, JN183897.1, DQ164195.1, JF357960.1, JF899530.1, JF899531.1, JN817352.1, JN891.1, JN183890.1,HQ671797.1, JF920759.1, EU391640.1, JX442282.1, AF260969.1, AJ 965628.1, KM488117.1, HM756678.1, FJ265702.1, FJ265703.1, HM756661.1, JX015519.1, JN183893.1, KC493970.1, AF394221.1, and AF196520.1. 


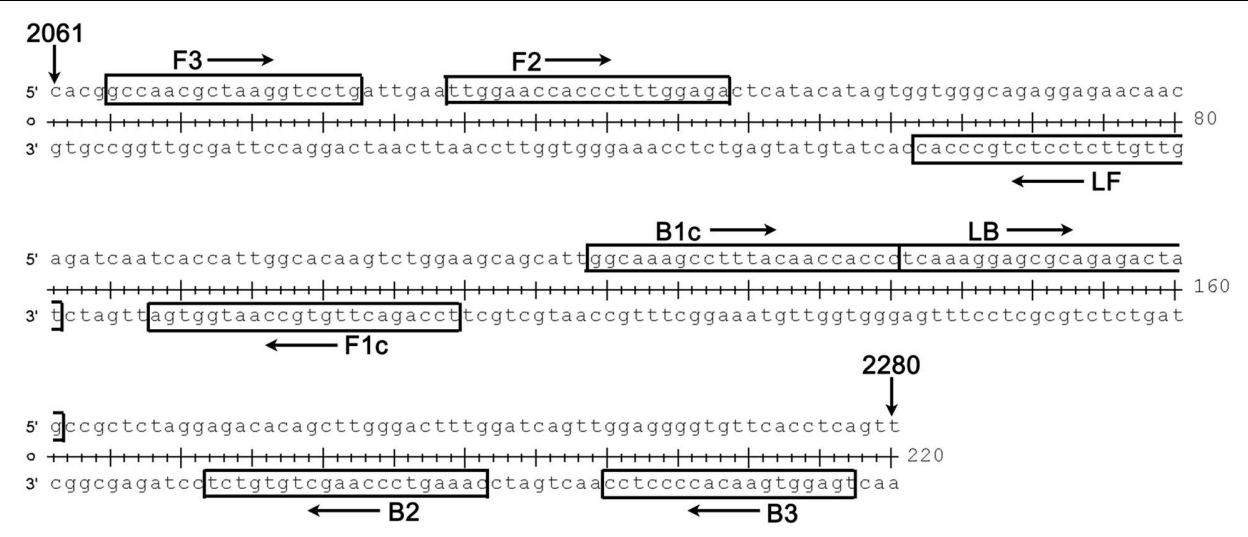

FIGURE 2 | Details (location and sequence) for each primer used in our RT-LAMP-VF assay. The target region spanned nucleotides $2065-2277$ of the complete genome of WNV strain NY99 (GenBank Accession: DQ211652); this region is located in the E gene of WNV.

TABLE 1 | Primer sequences used for the RT-LAMP-VF assay.

\begin{tabular}{lll}
\hline Primer name & Sequences $\left(\mathbf{5}^{\prime}\right.$ to $\left.\mathbf{3}^{\prime}\right)$ & Genome position \\
\hline LAMP-F3 & GCCAACGCTAAGGTCCTG & $2065-2082$ \\
LAMP-B3 & TGAGGTGAACACCCCTCC & $2260-2277$ \\
LAMP-FIP & TCCAGACTTGTGCCAATGGTGA & F1C:2148-2169 \\
$($ F1C + F2) & TTGGAACCACCCTTTGGAGA & F2:2089-2108 \\
LAMP-BIP & GGCAAAGCCTTTACAACCACCC & B1C:2179-2200 \\
$(B 1 C+$ B2) & CAAAGTCCCAAGCTGTGTCT & B2:2232-2251 \\
LAMP-LF & Biotin-TGTTGTTCTCCTCTGCCCAC & $2122-2141$ \\
LAMP-LB & FITC-TCAAAGGAGCGCAGAGACTAG & $2201-2221$
\end{tabular}

a The $5^{\prime}$ ends of the LAMP-LF was labeled with biotin. ${ }^{\mathrm{b}}$ The $5^{\prime}$ ends of the LAMP-LB was labeled with FITC.

polymerase large fragment (New England BioLabs), and $5 \mu \mathrm{l}$ of target RNA. The mixture was incubated at varying temperatures $\left(58,60,62,64\right.$, or $\left.66^{\circ} \mathrm{C}\right)$ for $60 \mathrm{~min}$, followed by heating at $80^{\circ} \mathrm{C}$ for $2 \mathrm{~min}$ to terminate the reaction. The mixture was incubated for different lengths of time $(30,40,50$ or $60 \mathrm{~min})$ at the optimal temperature. In parallel with the optimization of the reaction time, two different concentrations were used: one group of the six primers was used at the concentration described above, and the other group was used at half this concentration. The RT-LAMP products were detected using a VF visualization strip (Ustar Biotech Co., Ltd., Hangzhou, China) as previously described (Cui et al., 2012).

\section{Specificity and Sensitivity of the RT-LAMP-VF Assay}

RNAs of rRABV-WNVE $\left(10^{5} \mathrm{TCID}_{50} / \mathrm{ml}\right)$, JEV (10 $\left.5 \mathrm{PFU} / \mathrm{ml}\right)$, dengue virus (DENV, $\left.10^{5} \mathrm{PFU} / \mathrm{ml}\right)$, and classical swine fever virus (CSFV, $10^{5} \mathrm{TCID}_{50} / \mathrm{ml}$ ) were extracted using a commercial RNA extraction kit (RNeasy Mini Kit, Qiagen, Hilden, Germany). All operations were performed according to the manufacturer's instructions, and the level of extracted RNAs was determined as 80-120 ng/ $\mu \mathrm{l}$. To evaluate the specificity of the RT-LAMP-VF assay, $5 \mu$ l of RNAs extracted from above viruses and synthesized RNA transcripts $\left(10^{7}\right.$ copies/ $\left.\mu \mathrm{l}\right)$ were added into the optimal reaction system.
The synthesized WNV RNA transcripts (1092 ng/ $\mu \mathrm{l}$ ) were purified, quantified, and prepared as standard samples with a concentration of $10^{9}$ copies/ $\mu$ l. Then, 10 -fold serial dilutions of the standard samples (ranging from $10^{7}$ to $10^{0}$ copies/ $\mu \mathrm{l}$ ) were used to assess the detection limits of the RT-LAMP-VF assay.

\section{Evaluation of the RT-LAMP-VF Assay Using Live Virus RNA}

Viral RNA extracted from rRABV-WNVE was used to further evaluate the performance ability of the RT-LAMP-VF assay. rRABV-WNVE at a titer of $10^{6.5} \mathrm{TCID}_{50} / \mathrm{ml}$ was 10 -fold serial diluted in DMEM. RNA was extracted from the dilutions, and the RNA samples were detected using the RT-LAMP-VF assay.

\section{Evaluation of the RT-LAMP-VF Assay Using Clinical Specimens}

The feasibility of using the RT-LAMP-VF assay to detect WNV in clinical specimens was evaluated using 5-day-old and 3-weekold ICR mice. The mice were randomly divided into seven groups and intracerebrally infected with 10-fold dilutions of rRABV-WNVE ranging from $10^{6}$ to $10^{1} \mathrm{TCID}_{50}$ per mouse. DMEM was used as a placebo. At 7 days post-infection, the mice were submitted to humane euthanasia by cervical dislocation under ketamine-xylazine anesthesia at a dose of $0.1 \mathrm{~mL} / 10 \mathrm{~g}$ body weight, after which their brains were collected. The brain samples were then homogenized in phosphate-buffered saline (PBS, $10 \mathrm{mM}, \mathrm{pH}$ 7.2-7.4). The homogenates were centrifuged to remove debris, and the supernatants were collected for RNA extraction and virus titration.

Total RNA was extracted from the brain homogenate using an RNAeasy Mini Kit (Qiagen, Hilden, Germany). The extracted RNA was then assessed using the RT-LAMP-VF assay and realtime PCR as described previously (Yang et al., 2012). After viral titration, the brain homogenate with a high titer of $7.03 \mathrm{Log}$ (TCID $_{50} / g$ tissue) was selected to evaluate the sensitivity of the assay. To accomplish this, RNA was extracted from a 10-fold serial dilution of the brain homogenate and measured using the 
RT-LAMP-VF assay; brain homogenate from healthy mice was used as a control.

\section{Ethics Statement}

All animal studies performed in this work were approved by the Animal Care and Use Committee of the Chinese People's Liberation Army (No. SYXK2009-045). All volunteers involved in this study provided written informed consent for the use of blood samples. All efforts were made to minimize animal suffering.

\section{RESULTS}

\section{Product Detection Using the RT-LAMP-VF Assay}

When using the VF visualization strip, a clearly visible red-purple band at the control line was necessary for the test to be considered valid. The appearance of two red-purple bands at both the test and control lines was regarded as a positive result. If only the band at the control line appeared, then the result was considered negative (Figure 3).

\section{Optimization of RT-LAMP-VF Reaction Conditions}

To determine the optimal conditions for the RT-LAMP-VF assay, synthesized RNA transcripts were used as a template to optimize both assay temperature and assay time. To determine the optimal temperature for the assay, $60-\mathrm{min}$ reactions were performed at five different temperatures $\left(58,60,62,64\right.$, and $\left.66^{\circ} \mathrm{C}\right)$. The results indicated that running the assay at $64^{\circ} \mathrm{C}$ produced the strongest amplification signal (Table 2); therefore, $64^{\circ} \mathrm{C}$ was considered the optimal temperature for the assay.

To determine the optimal duration of time required for the RT-LAMP-VF assay, four different reaction times (30, 40, 50, or $60 \mathrm{~min}$ ) were compared at $64^{\circ} \mathrm{C}$. The best sensitivity and

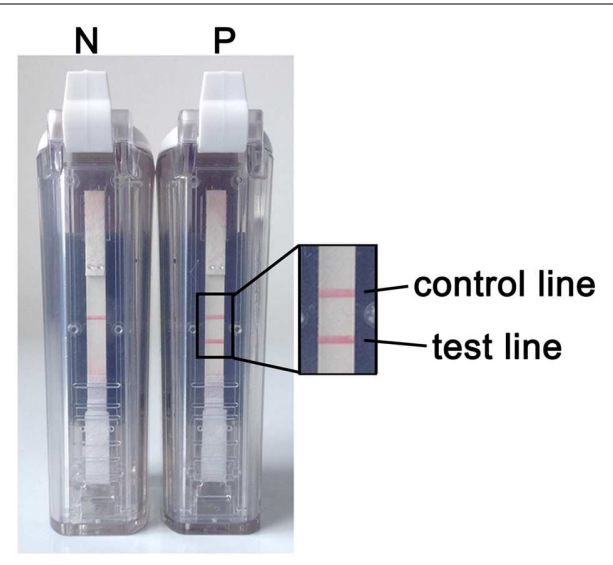

FIGURE 3 | RT-LAMP-VF assay results. $N$ denotes a negative result. In this case, only one red-purple band appears at the control line. $P$ denotes a positive result. In this case, two red-purple bands appear: one at the test line and one at the control line.
TABLE 2 | Reaction temperature optimization for RT-LAMPa .

\begin{tabular}{lccccccccc}
\hline Temperature $/{ }^{\circ} \mathbf{C}$ & \multicolumn{7}{c}{ RNA dilution (Copies/ $\mu \mathrm{l})$} \\
\cline { 2 - 8 } & $\mathbf{1 0}^{\mathbf{7}}$ & $\mathbf{1 0}^{\mathbf{6}}$ & $\mathbf{1 0 ^ { 5 }}$ & $\mathbf{1 0}^{\mathbf{4}}$ & $\mathbf{1 0}^{3}$ & $\mathbf{1 0}^{2}$ & $\mathbf{1 0}^{\mathbf{1}}$ & $\mathbf{1 0}^{\mathbf{0}}$ \\
\hline 58 & + & + & + & + & - & - & - & - \\
60 & + & + & + & + & - & - & - & - \\
62 & + & + & + & + & $+^{\mathrm{b}}$ & - & - & - \\
64 & + & + & + & + & + & - & - & - \\
66 & + & + & + & + & - & - & - & - \\
\hline
\end{tabular}

a Three replications were performed for each trial. ${ }^{\mathrm{b}}$ The result was weakly positive.

specificity were found when the reaction lasted for $40 \mathrm{~min}$ at $64^{\circ} \mathrm{C}$ (Table 3). Therefore, an amplification time of $40 \mathrm{~min}$ was selected as the optimal time for the assay.

As shown in Table 3, primer concentration was also optimized when optimizing the reaction time. The following primer concentrations were considered optimal: $0.4 \mu \mathrm{M}$ for the inner primers FIP and BIP, $0.2 \mu \mathrm{M}$ for the loop primers LF and LB, and $0.1 \mu \mathrm{M}$ for the outer primers $\mathrm{F} 3$ and $\mathrm{B} 3$.

\section{Specificity of the RT-LAMP-VF Assay}

The analytical specificity of the RT-LAMP-VF assay was determined using synthesized RNA transcripts and RNA extracted from control viruses, including JEV, DENV, and CSFV. As shown in Figure 4, all of the tested control samples appeared identical to the blank control and produced only one red-purple band at the location of the control line; therefore, all of these samples produced negative test results. A positive test line was observed when using synthetic WNV RNA transcripts and RNA extracted from rRABV-WNVE as templates. Thus, because the RT-LAMP-VF assay had no cross-reactivity with other viruses related to $\mathrm{WNV}$, the assay was considered to have high specificity.

\section{Sensitivity of the RT-LAMP-VF Assay}

Synthetic WNV RNA transcripts were 10 -fold serially diluted to create samples ranging in concentration from $10^{7}$ to $10^{0}$ copies

\begin{tabular}{|c|c|c|c|c|c|c|c|c|c|}
\hline \multirow{2}{*}{$\begin{array}{l}\text { Time/ } \\
\text { min }\end{array}$} & \multirow{2}{*}{$\begin{array}{l}\text { Primers } \\
\text { group }\end{array}$} & \multicolumn{8}{|c|}{ RNA dilution (Copies/ $\mu \mathrm{l}$ ) } \\
\hline & & $10^{7}$ & $10^{6}$ & $10^{5}$ & $10^{4}$ & $10^{3}$ & $10^{2}$ & $10^{1}$ & $10^{\circ}$ \\
\hline \multirow[t]{2}{*}{30} & $1^{b}$ & + & + & $+^{d}$ & $+^{d}$ & - & - & - & - \\
\hline & $2^{c}$ & + & + & + & + & - & - & - & - \\
\hline \multirow[t]{2}{*}{40} & 1 & + & + & + & + & + & + & - & - \\
\hline & 2 & + & + & + & + & - & - & - & - \\
\hline \multirow[t]{2}{*}{50} & 1 & + & + & + & + & + & - & - & - \\
\hline & 2 & + & + & + & + & - & - & - & - \\
\hline \multirow[t]{2}{*}{60} & 1 & + & + & + & $+^{d}$ & - & - & - & - \\
\hline & 2 & + & + & + & + & + & - & - & - \\
\hline
\end{tabular}

a Three replications were performed for each trial. ${ }^{\mathrm{b}}$ The six primers was used as described as bellow: $0.4 \mu \mathrm{M}$ each of inner primers FIP and BIP, $0.2 \mu \mathrm{M}$ loop primers $L F$ and $L B, 0.1 \mu M$ outer primers F3 and B3. ${ }^{c} T$ The six primers was used as described as bellow: $0.2 \mu \mathrm{M}$ each of inner primers FIP and BIP, $0.1 \mu \mathrm{M}$ loop primers $L F$ and $L B, 0.05 \mu M$ outer primers $F 3$ and B3. dThe result was weakly positive. 


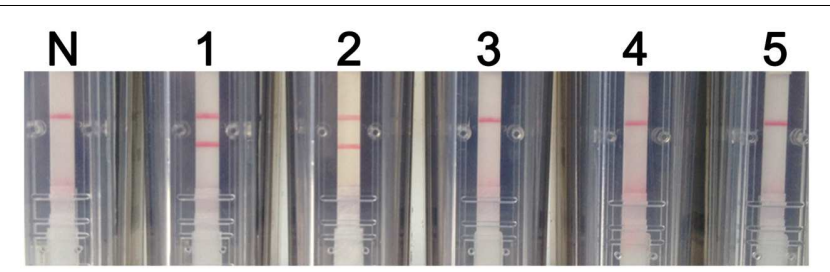

FIGURE 4 | RT-LAMP-VF assay specificity. The specificity of the RT-LAMP-VF assay was determined by analyzing synthesized RNA transcripts and RNA extracted from rRABV-WNVE, JEV, DENV, and CSFV. N: blank control; 1: synthesized RNA transcripts; 2: rRABV-WNVE RNA; 3: JEV RNA; 4: DENV RNA; 5: CSFV RNA.

per $\mu l$. These samples were then used to assess the sensitivity of the RT-LAMP-VF assay. After a 40-min amplification reaction, negative results were produced at concentrations lower than $10^{2}$ copies/ $\mu$ l. Thus, the RT-LAMP-VF assay had a detection limit of $10^{2}$ copies/ $\mu 1$ of synthetic RNA (Figure 5).

\section{Evaluation of the RT-LAMP-VF Assay Using Live Virus RNA}

Total RNA was extracted from serial dilutions of rRABV-WNVE and then assessed using the RT-LAMP-VF assay. As shown in Figure 6, RNA extracted from a $10^{5}$-fold dilution of virus $\left(10^{1.5}\right.$ $\mathrm{TCID}_{50} / \mathrm{ml}$ ) produced positive results when assayed; thus, the detection limit of the assay for rRABV-WNVE in cell culture is $10^{1.5} \mathrm{TCID}_{50} / \mathrm{ml}$. To confirm the absence of non-specific reactions, total RNA extracted from DMEM and NA cells was also evaluated; no amplification was found in either case.

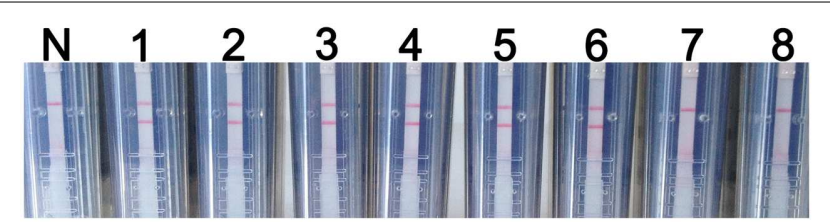

FIGURE 5 | RT-LAMP-VF assay sensitivity. The sensitivity of the RT-LAMP-VF assay was analyzed by using tenfold serial dilutions of synthesized RNA transcripts. N: blank control; $1: 10^{7}$ copies/ $\mu$ l; 2: $10^{6}$ copies/ $\mu$ l; 3: $10^{5}$ copies/ $\mu$ l; 4: $10^{4}$ copies $/ \mu$ l; $5: 10^{3}$ copies $/ \mu l ; 6: 10^{2}$

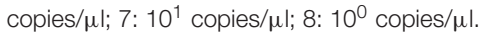

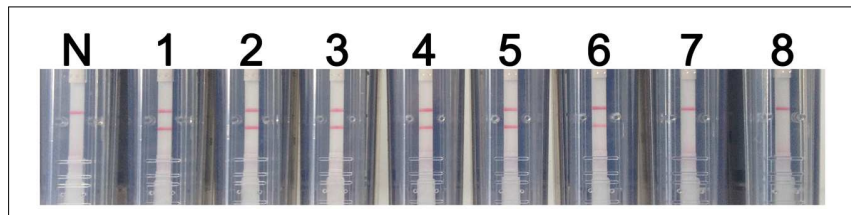

FIGURE 6 | Evaluation of the RT-LAMP-VF assay using live virus. The sensitivity of the RT-LAMP-VF assay was analyzed by using 10-fold serial dilutions of recombinant virus (rRABV-WNVE). N: blank control; $1: 10^{6.5}$ $\mathrm{TCID}_{50} / \mathrm{ml} ; 2: 10^{5.5} \mathrm{TCID}_{50} / \mathrm{ml} ; 3: 10^{4.5} \mathrm{TCID}_{50} / \mathrm{ml} ; 4: 10^{3.5} \mathrm{TCID}_{50} / \mathrm{ml}$; 5: $10^{2.5} \mathrm{TCID}_{50} / \mathrm{ml} ; 6: 10^{1.5} \mathrm{TCID}_{50} / \mathrm{ml} ; 7: 10^{0.5} \mathrm{TCID}_{50} / \mathrm{ml} ; 8: 10^{-0.5}$ $\mathrm{TClD}_{50} / \mathrm{ml}$.

\section{Evaluation of the RT-LAMP-VF Assay Using Clinical Specimens}

Brain tissues collected from mice infected with rRABV-WNVE were used as clinical specimens to further evaluate the RTLAMP-VF assay. As shown in Table 4, rRABV-WNVE mRNA in the range of 6.14-10.02 $\log$ (copy number/g tissue) was extracted from the brains of rRABV-WNVE-infected suckling mice, corresponding to viral titers in the range of 4.31-7.35 $\log \left(\mathrm{TCID}_{50} / \mathrm{g}\right.$ tissue); the RT-LAMP-VF results were positive across this entire range. Unlike the suckling mice, substantially less $\mathrm{rRABV}$-WNVE mRNA was extracted from the brains of 3-week-old mice brain: from 2.16 to $5.56 \log$ (copy number/g tissue) mRNA was extracted, and two of the samples from the $10^{2}$ group were not detected. Thus, viral titers could not be determined for the 3-week-old mice; however, all of the RTLAMP-VF results were positive, except for three samples in the $10^{1}$ and $10^{2}$ groups (Table 5).

The sensitivity of the RT-LAMP-VF assay was further evaluated using brain homogenates collected from rRABVWNVE-infected mice; brain homogenates from uninfected mice were used as a control. After diluting the rRABV-WNVE-infected

TABLE 4 | Assay detection results from suckling mouse brain tissue samples.

\begin{tabular}{|c|c|c|c|c|}
\hline \multicolumn{2}{|c|}{ Samples $^{a}$} & $\begin{array}{l}\text { mRNA [Log (copy } \\
\text { number/g tissue)] }\end{array}$ & $\begin{array}{l}\text { Virus titers [Log } \\
\text { (TCID }_{50} / g \text { tissue)] }\end{array}$ & RT-LAMP \\
\hline \multicolumn{2}{|c|}{ Blank } & Not detected & Not detected & - \\
\hline \multicolumn{2}{|c|}{ Negative 1} & Not detected & Not detected & - \\
\hline \multicolumn{2}{|c|}{ Negative 2} & Not detected & Not detected & - \\
\hline \multirow[t]{4}{*}{$10^{1}$} & 1 & 8.05 & 5.32 & + \\
\hline & 2 & 7.21 & 6.67 & + \\
\hline & 3 & 9.59 & 5.67 & + \\
\hline & 4 & 6.14 & 6.66 & + \\
\hline \multirow[t]{4}{*}{$10^{2}$} & 1 & 9.29 & 6.15 & + \\
\hline & 2 & 10.02 & 7.03 & + \\
\hline & 3 & 9.28 & 7.27 & + \\
\hline & 4 & 7.30 & 6.18 & + \\
\hline \multirow[t]{4}{*}{$10^{3}$} & 1 & 8.93 & 5.46 & + \\
\hline & 2 & 8.46 & 6.24 & + \\
\hline & 3 & 8.14 & 6.33 & + \\
\hline & 4 & 7.65 & 5.68 & + \\
\hline \multirow[t]{4}{*}{$10^{4}$} & 1 & 7.58 & 6.18 & + \\
\hline & 2 & 9.45 & 5.09 & + \\
\hline & 3 & 7.35 & 7.21 & + \\
\hline & 4 & 6.51 & 6.16 & + \\
\hline \multirow[t]{4}{*}{$10^{5}$} & 1 & 8.96 & 6.62 & + \\
\hline & 2 & 8.79 & 4.31 & + \\
\hline & 3 & 7.48 & 7.35 & + \\
\hline & 4 & 7.17 & 7.34 & + \\
\hline \multirow[t]{4}{*}{$10^{6}$} & 1 & 8.38 & 5.22 & + \\
\hline & 2 & 8.28 & 6.65 & + \\
\hline & 3 & 9.27 & 4.35 & + \\
\hline & 4 & 8.82 & 6.23 & + \\
\hline
\end{tabular}

a $10^{1}, 10^{2}, 10^{3}, 10^{4}, 10^{5}, 10^{6}$ are the virus dose which was infected in mice (TCID 50 /mouse); 1, 2, 3 are different numbers of mouse in each group. 
TABLE 5 | Assay detection results from 3-week-old mouse brain tissue samples.

\begin{tabular}{|c|c|c|c|c|}
\hline \multicolumn{2}{|c|}{ Samples $^{a}$} & $\begin{array}{l}\text { mRNA [Log (copy } \\
\text { number/g tissue)] }\end{array}$ & $\begin{array}{l}\text { Virus titers [Log } \\
\text { (TCID }_{50} / g \text { tissue)] }\end{array}$ & RT-LAMP \\
\hline \multicolumn{2}{|c|}{ Blank } & Not detected & Not detected & - \\
\hline \multicolumn{2}{|c|}{ Negative 1} & Not detected & Not detected & - \\
\hline \multicolumn{2}{|c|}{ Negative 2} & Not detected & Not detected & - \\
\hline \multirow[t]{3}{*}{$10^{1}$} & 1 & 2.36 & Not detected & + \\
\hline & 2 & 4.11 & Not detected & + \\
\hline & 3 & 2.16 & Not detected & - \\
\hline \multirow[t]{3}{*}{$10^{2}$} & 1 & Not detected & Not detected & - \\
\hline & 2 & Not detected & Not detected & - \\
\hline & 3 & 2.49 & Not detected & + \\
\hline \multirow[t]{3}{*}{$10^{3}$} & 1 & 4.73 & Not detected & + \\
\hline & 2 & 3.02 & Not detected & + \\
\hline & 3 & 5.11 & Not detected & + \\
\hline \multirow[t]{3}{*}{$10^{4}$} & 1 & 3.23 & Not detected & + \\
\hline & 2 & 5.76 & Not detected & + \\
\hline & 3 & 3.52 & Not detected & + \\
\hline \multirow[t]{3}{*}{$10^{5}$} & 1 & 3.53 & Not detected & + \\
\hline & 2 & 4.00 & Not detected & + \\
\hline & 3 & 4.13 & Not detected & + \\
\hline \multirow[t]{3}{*}{$10^{6}$} & 1 & 4.68 & Not detected & + \\
\hline & 2 & 4.97 & Not detected & + \\
\hline & 3 & 5.56 & Not detected & + \\
\hline
\end{tabular}

a $10^{1}, 10^{2}, 10^{3}, 10^{4}, 10^{5}, 10^{6}$ are the virus dose which was infected in mice (TCID 50 /mouse); 1, 2, 3, 4 are different numbers of mouse in each group.

brain homogenates by $10^{5}$-fold, the RT-LAMP-VF assay still produced positive results. Therefore, the sensitivity of the assay for rRABV-WNV-infected mouse brain tissue is $10^{1.33}$ $\mathrm{TCID}_{50} / \mathrm{ml}$.

\section{DISCUSSION}

With the unexpected appearance of WNV in many countries, it is becoming increasingly important to develop adequate surveillance methods for WNV. Such methods are needed not only in countries where WNV infections are epidemic but also in countries threatened by WNV infection and even in countries where WNV infection has not yet spread. In addition, the development of a rapid and reliable method for diagnosing WNV is critically important for the prevention of infection, the implementation of appropriate countermeasures, and the optimization of healthcare resources. In the current study, we described the development of a RT-LAMP-VF assay that provides a simple and rapid method to detect WNV infection.

To accomplish the above, we analyzed the complete genome sequences of 30 strains of WNV isolated over various years from different regions and disparate species, including representative strains from WNV lineages 1, 2, and 3. Bases on this analysis, we selected two target regions for RT-LAMP-VF, a region located in the E gene and a region located in the $5^{\prime}$-UTR and spanning part of the $\mathrm{C}$ gene. However, when using the RT-LAMP-VF assay to detect the latter region, the assay produced low sensitivity (data not shown). Several previously reported molecular biology methods have used the E gene as a target sequence with excellent results, consistent with our previous study (Parida et al., 2004; Zink et al., 2013; Kumar et al., 2014). Therefore, we chose a relatively conserved region of the genome spanning nucleotide positions 2065-2277 as a target sequence.

We designed a set of six primers (including two loop primers) targeting eight regions located in the conserved region of the WNV E gene to ensure high specificity for nucleic acid amplification. The use of loop primers accelerated the reaction time, enabling the reaction to be completed in less than half the time required for our original LAMP assay (Nagamine et al., 2002). Indeed, amplification could be completed within $40 \mathrm{~min}$, which is faster than other molecular biology methods.

Compared to other molecular biology detection technologies, the RT-LAMP-VF assay is economical, technically simple, and rapid. For this assay, reverse transcription and cDNA amplification are performed in a single step, and no additional procedures are needed. Moreover, some conventional molecular biology methods, such as RT-PCR, nested RT-PCR and realtime PCR, have inherent flaws; in particular, specialized PCR instruments are needed, and temperature must be precisely controlled for a lengthy period of time. This inhibits the application of these methods in rural and remote areas (Deng et al., 2015). As the reaction conditions for RT-LAMP-VF are isothermal, only a water bath is needed to complete the assay, offering a measure of practicality for field laboratories in economically impoverished areas.

Parida et al. (2004) developed a RT-LAMP method for the rapid detection of WNV. In this method, the results were analyzed using agarose gel electrophoresis or real-time turbidity analysis (Parida et al., 2004). Generally, the use of gel electrophoresis to detect amplification products increases the risk of product contamination and degradation, while real-time turbidity analysis suffers from background interference (Iwamoto et al., 2003; Ge et al., 2013). In the current study, we modified the RT-LAMP technique by using two loop primers labeled with FITC and biotin. This allowed labeled amplification products to be analyzed using a VF visualization strip housed inside of an enclosed, plastic, leak-proof device, without the need for an electrophoresis apparatus or a turbidimeter. Within the strip, the FITC-labeled amplification products can bind to the antiFITC antibody located on the test line, and the biotin-labeled amplification products are captured by colloidal gold particles conjugated to the anti-biotin antibodies (Chow et al., 2008; Cui et al., 2012). Using this strategy, we reduced not only the reaction time but also the chances of product contamination. Moreover, the results of the assay can be directly visualized within $2 \mathrm{~min}$, and the plastic device was designed to prevent leakage of the amplification products.

As encephalitis is the main clinical symptom of WNV infection, we used brain samples from Charadrius alexandrinus and Recurvirostra avosetta to evaluate the specificity of the RT-LAMP-VF assay. All of the samples were negative (data not shown). Clinical specimens infected with WNV (e.g., blood and brain tissue) are difficult to obtain in non-endemic countries such as China; however, we hypothesized that using 
a recombinant virus expressing the $\mathrm{E}$ gene of WNV would produce similar assay results. Therefore, we assessed brain tissues collected from rRABV-WNVE-infected mice as clinical specimens in this study. Our results indicated that the assay could successfully detect clinical samples infected with the recombinant virus. Additionally, the assay thresholds for detecting cell culture samples infected with the recombinant virus (sensitivity: $10^{1.5} \mathrm{TCID}_{50} / \mathrm{ml}$ ) and mouse brain tissues infected with the recombinant virus (sensitivity: $10^{1.33} \mathrm{TCID}_{50} / \mathrm{ml}$ ) were nearly identical, which indicated that the presence of tissue-specific RNA did not influence the performance of the RT-LAMP-VF assay. Furthermore, three of the samples collected from the 3week-old ICR mice had very little rRABV-WNVE mRNA, and the RT-LAMP-VF results were negative for these samples. This result indicates that the assay's results may be ambiguous in cases of very small viral loads. Under such conditions, sample detection should be performed in duplicate or combined with other methods. To simulate brief viremia, blood samples from healthy volunteers were mixed with rRABV-WNVE and evaluated using the RTLAMP-VF assay. In this case, similar results were produced as those generated by the rRABV-WNVE-infected mouse brain tissues (data not shown). The broad capacity to accurately identify the presence of WNVE in clinical specimens validated the RT-LAMP-VF assay for the detection of WNV infection.

In summary, we developed a method to diagnose WNV using isothermal amplification combined with a VF visualization

\section{REFERENCES}

Brinton, M. A. (2002). The molecular biology of West Nile Virus: a new invader of the western hemisphere. Annu. Rev. Microbiol. 56, 371-402. doi: 10.1146/annurev.micro.56.012302.160654

Caraballo, E. V., Hunsperger, E., and Martinez, I. (2015). Characterization of puerto rican west nile virus isolates in mice. Virol. J. 12:137. doi: 10.1186/s12985-015-0363-8

Chow, W. H., McCloskey, C., Tong, Y., Hu, L., You, Q., Kelly, C. P., et al. (2008). Application of isothermal helicase-dependent amplification with a disposable detection device in a simple sensitive stool test for toxigenic Clostridium difficile. J. Mol. Diagn. 10, 452-458. doi: 10.2353/jmoldx.2008. 080008

Cui, L., Ge, Y., Qi, X., Xu, G., Li, H., Zhao, K., et al. (2012). Detection of severe fever with thrombocytopenia syndrome virus by reverse transcription-cross-priming amplification coupled with vertical flow visualization. J. Clin. Microbiol. 50, 3881-3885. doi: 10.1128/JCM.01931-12

Dauphin, G., and Zientara, S. (2007). West Nile virus: recent trends in diagnosis and vaccine development. Vaccine 25, 5563-5576. doi: 10.1016/j.vaccine.2006.12.005

De Filette, M., Ulbert, S., Diamond, M., and Sanders, N. N. (2012). Recent progress in West Nile virus diagnosis and vaccination. Vet. Res. 43:16. doi: 10.1186/1297-9716-43-16

Del Amo, J., Sotelo, E., Fernandez-Pinero, J., Gallardo, C., Llorente, F., Aguero, M., et al. (2013). A novel quantitative multiplex real-time RTPCR for the simultaneous detection and differentiation of West Nile virus lineages 1 and 2, and of Usutu virus. J. Virol. Methods 189, 321-327. doi: 10.1016/j.jviromet.2013.02.019

Deng, J., Pei, J., Gou, H., Ye, Z., Liu, C., and Chen, J. (2015). Rapid and simple detection of Japanese encephalitis virus by reverse transcription loop-mediated isothermal amplification combined with a lateral flow dipstick. J. Virol. Methods 213, 98-105. doi: 10.1016/j.jviromet.2014.12.006

Deubel, V., Fiette, L., Gounon, P., Drouet, M. T., Khun, H., Huerre, M., et al. (2001). Variations in biological features of West Nile viruses. Ann. N. Y. Acad. Sci. 951, 195-206. doi: 10.1111/j.1749-6632.2001.tb02697.x strip. This method was shown to have high specificity and high sensitivity for the detection of WNV. To simulate clinical samples, brain tissue infected with a recombinant virus expressing the $\mathrm{E}$ gene of $\mathrm{WNV}$ was evaluated. The results showed that the assay could accurately detect the virus with no interference from tissue-specific RNA. Furthermore, in the clinic, viral RNA loads reach higher levels than the detection limit of our assay (Ramos et al., 2012; Caraballo et al., 2015). Collectively, the above results demonstrate that our RT-LAMP-VF assay offers rapid, simple and effective diagnostic identification of WNV infection.

\section{AUTHOR CONTRIBUTIONS}

HW, SY, and XX designed the experiments. ZC, LW, LL, HJ, CX, FY, JW, QL, YZ, and TW performed the experiment. ZC, HW, LL, YG, YL, SY, and XX analyzed the data. ZC and HW wrote the manuscript. All authors reviewed the manuscript.

\section{ACKNOWLEDGMENT}

This work was supported in part by the military medical health project grant (NO. 13CXZ024) and National Key Technologies R\&D Program (No. 2013BAD12B04).

Faggioni, G., De Santis, R., Pomponi, A., Fantini, M., Savini, G., Monaco, F., et al. (2014). Rapid molecular detection and genotyping of West Nile Virus lineages 1 and 2 by real time PCR and melting curve analysis. J. Virol. Methods 207, 54-59. doi: 10.1016/j.jviromet.2014.06.020

Ge, Y., Wu, B., Qi, X., Zhao, K., Guo, X., Zhu, Y., et al. (2013). Rapid and sensitive detection of novel avian-origin influenza A (H7N9) virus by reverse transcription loop-mediated isothermal amplification combined with a lateralflow device. PLoS ONE 8:e69941. doi: 10.1371/journal.pone.0069941

Gubler, D. J. (2001). Human arbovirus infections worldwide. Ann. N. Y. Acad. Sci. 951, 13-24. doi: 10.1111/j.1749-6632.2001.tb02681.x

Hayes, E. B., and Gubler, D. J. (2006). West Nile virus: epidemiology and clinical features of an emerging epidemic in the United States. Annu. Rev. Med. 57, 181-194. doi: 10.1146/annurev.med.57.121304.131418

Hayes, E. B., and O'Leary, D. R. (2004). West Nile virus infection: a pediatric perspective. Pediatrics 113, 1375-1381. doi: 10.1542/peds.113.5.1375

Iwamoto, T., Sonobe, T., and Hayashi, K. (2003). Loop-mediated isothermal amplification for direct detection of Mycobacterium tuberculosis complex, M. avium, and $M$. intracellulare in sputum samples. J. Clin. Microbiol. 41, 2616-2622. doi: 10.1128/JCM.41.6.2616-2622.2003

Kumar, J. S., Saxena, D., and Parida, M. (2014). Development and comparative evaluation of SYBR Green I-based one-step real-time RT-PCR assay for detection and quantification of West Nile virus in human patients. Mol. Cell. Probes 28, 221-227. doi: 10.1016/j.mcp.2014.03.005

Kurosaki, Y., Takada, A., Ebihara, H., Grolla, A., Kamo, N., Feldmann, H., et al. (2007). Rapid and simple detection of Ebola virus by reverse transcriptionloop-mediated isothermal amplification. J. Virol. Methods 141, 78-83. doi: 10.1016/j.jviromet.2006.11.031

Lanciotti, R. S., Ebel, G. D., Deubel, V., Kerst, A. J., Murri, S., Meyer, R., et al. (2002). Complete genome sequences and phylogenetic analysis of West Nile virus strains isolated from the United States, Europe, and the Middle East. Virology 298, 96-105. doi: 10.1006/viro.2002.1449

Lim, S. M., Koraka, P., Osterhaus, A. D., and Martina, B. E. (2011). West Nile virus: immunity and pathogenesis. Viruses 3, 811-828. doi: 10.3390/v3060811

Mikawa, A. Y., Santos, S. A., Kenfe, F. R., da Silva, F. H., and da Costa, P. I. (2009). Development of a rapid one-step immunochromatographic 
assay for HCV core antigen detection. J. Virol. Methods 158, 160-164. doi: 10.1016/j.jviromet.2009.02.013

Mukhopadhyay, S., Kim, B. S., Chipman, P. R., Rossmann, M. G., and Kuhn, R. J. (2003). Structure of West Nile virus. Science 302, 248. doi: $10.1126 /$ science. 1089316

Nagamine, K., Hase, T., and Notomi, T. (2002). Accelerated reaction by loopmediated isothermal amplification using loop primers. Mol. Cell. Probes 16, 223-229. doi: 10.1006/mcpr.2002.0415

Nash, D., Mostashari, F., Fine, A., Miller, J., O’Leary, D., Murray, K., et al. (2001). The outbreak of West Nile virus infection in the New York City area in 1999. N. Engl. J. Med. 344, 1807-1814. doi: 10.1056/NEJM200106143442401

Notomi, T., Okayama, H., Masubuchi, H., Yonekawa, T., Watanabe, K., Amino, N., et al. (2000). Loop-mediated isothermal amplification of DNA. Nucleic Acids Res. 28:E63. doi: 10.1093/nar/28.12.e63

Parida, M., Posadas, G., Inoue, S., Hasebe, F., and Morita, K. (2004). Real-time reverse transcription loop-mediated isothermal amplification for rapid detection of West Nile virus. J. Clin. Microbiol. 42, 257-263. doi: 10.1128/JCM.42.1.257-263.2004

Ramos, H. J., Lanteri, M. C., Blahnik, G., Negash, A., Suthar, M. S., Brassil, M. M., et al. (2012). IL-1beta signaling promotes CNS-intrinsic immune control of West Nile virus infection. PLoS Pathog. 8:e1003039. doi: 10.1371/journal.ppat.1003039

Sejvar, J. J., Haddad, M. B., Tierney, B. C., Campbell, G. L., Marfin, A. A., Van Gerpen, J. A., et al. (2003). Neurologic manifestations and outcome of West Nile virus infection. JAMA 290, 511-515. doi: 10.1001/jama.290.4.511

Solomon, T., Ooi, M. H., Beasley, D. W., and Mallewa, M. (2003). West Nile encephalitis. BMJ 326, 865-869. doi: 10.1136/bmj.326.7394.865

Vazquez, A., Sanchez-Seco, M. P., Ruiz, S., Molero, F., Hernandez, L., Moreno, J., et al. (2010). Putative new lineage of west nile virus. Spain. Emerg. Infect. Dis. 16, 549-552. doi: 10.3201/eid1603.091033
Wang, H., Feng, N., Yang, S., Wang, C., Wang, T., Gao, Y., et al. (2010). A rapid immunochromatographic test strip for detecting rabies virus antibody. J. Virol. Methods 170, 80-85. doi: 10.1016/j.jviromet.2010.09.002

Wang, H., Zhang, G., Wen, Y., Yang, S., Xia, X., and Fu, Z. F. (2011). Intracerebral administration of recombinant rabies virus expressing GM-CSF prevents the development of rabies after infection with street virus. PLoS ONE 6:e25414. doi: 10.1371/journal.pone.0025414

Whiting, S. H., and Champoux, J. J. (1998). Properties of strand displacement synthesis by Moloney murine leukemia virus reverse transcriptase: mechanistic implications. J. Mol. Biol. 278, 559-577. doi: 10.1006/jmbi.1998. 1720

Yang, Y. J., Zhao, P. S., Zhang, T., Wang, H. L., Liang, H. R., Zhao, L. L., et al. (2012). Small interfering RNAs targeting the rabies virus nucleoprotein gene. Virus Res. 169, 169-174. doi: 10.1016/j.virusres.2012.07.024

Zink, S. D., Jones, S. A., Maffei, J. G., and Kramer, L. D. (2013). Quadraplex qRT-PCR assay for the simultaneous detection of Eastern equine encephalitis virus and West Nile virus. Diagn. Microbiol. Infect. Dis. 77, 129-132. doi: 10.1016/j.diagmicrobio.2013.06.019

Conflict of Interest Statement: The authors declare that the research was conducted in the absence of any commercial or financial relationships that could be construed as a potential conflict of interest.

Copyright (C) 2016 Cao, Wang, Wang, Li, Jin, Xu, Feng, Wang, Li, Zhao, Wang, Gao, Lu, Yang and Xia. This is an open-access article distributed under the terms of the Creative Commons Attribution License (CC BY). The use, distribution or reproduction in other forums is permitted, provided the original author(s) or licensor are credited and that the original publication in this journal is cited, in accordance with accepted academic practice. No use, distribution or reproduction is permitted which does not comply with these terms. 